\title{
High-Efficiency Volume Reflection of an Ultrarelativistic Proton Beam with a Bent Silicon Crystal
}

\author{
Walter Scandale \\ CERN, European Organization for Nuclear Research, CH-1211 Geneva 23, Switzerland \\ Dean A. Still \\ Fermi National Accelerator Laboratory, P.O. Box 500, Batavia, Illinois 60510-0500, USA
}

\begin{abstract}
Alberto Carnera, ${ }^{1,2}$ Gianantonio Della Mea, ${ }^{1,3}$ Davide De Salvador, ${ }^{1,2}$ Riccardo Milan, ${ }^{1}$ and Alberto Vomiero ${ }^{1,4}$
${ }^{1}$ INFN Laboratori Nazionali di Legnaro, Viale Università 2, 35020 Legnaro (PD), Italy

${ }^{2}$ Dipartimento di Fisica, Università di Padova, Via Marzolo 8, 35131 Padova, Italy

${ }^{3}$ Dipartimento di Ingegneria dei Materiali e Tecnologie Industriali, Università di Trento, Via Mesiano 77, 38050 Trento, Italy

${ }^{4}$ INFM-CNR, Via Valotti 9, 25133 Brescia, Italy

Stefano Baricordi, Pietro Dalpiaz, Massimiliano Fiorini, Vincenzo Guidi, Giuliano Martinelli, Andrea Mazzolari, and Emiliano Milan

INFN Sezione di Ferrara, Dipartimento di Fisica, Università di Ferrara, Via Saragat 1, 44100 Ferrara, Italy

Giovanni Ambrosi, Philipp Azzarello, Roberto Battiston, Bruna Bertucci, William J. Burger, Maria Ionica, and Paolo Zuccon

INFN Sezione di Perugia and Università degli Studi di Perugia, Dipartimento di Fisica, Via Pascoli, 06123 Perugia, Italy

Gianluca Cavoto, Roberta Santacesaria, and Paolo Valente

INFN Sezione di Roma, Piazzale Aldo Moro 2, 00185 Rome, Italy

Erik Vallazza

INFN Sezione di Trieste, Via Valerio 2, 34127 Trieste, Italy
\end{abstract}

Alexander G. Afonin, Vladimir T. Baranov, Yury A. Chesnokov, Vladilen I. Kotov, Vladimir A. Maisheev, and Igor A. Yaznin

Institute of High Energy Physics, Moscow Region, RU-142284 Protvino, Russia

Sergey V. Afansiev, Alexander D. Kovalenko, and Alexander M. Taratin

Joint Institute for Nuclear Research, Joliot-Curie 6, 141980, Dubna, Moscow Region, Russia

Alexander S. Denisov, Yury A. Gavrikov, Yuri M. Ivanov, Vladimir G. Ivochkin, Sergey V. Kosyanenko, Anatoli A. Petrunin, Vyacheslav V. Skorobogatov, and Vsevolod M. Suvorov

Petersburg Nuclear Physics Institute, 188300 Gatchina, Leningrad Region, Russia

Davide Bolognini, Luca Foggetta, Said Hasan, and Michela Prest

Università dell'Insubria, via Valleggio 11, 22100 Como, Italy, and INFN Sezione di Milano, via Celoria 16, 20133 Milan, Italy

(Received 21 December 2006; published 9 April 2007)

\begin{abstract}
The volume reflection phenomenon was detected while investigating $400 \mathrm{GeV}$ proton interactions with bent silicon crystals in the external beam H8 of the CERN Super Proton Synchrotron. Such a process was observed for a wide interval of crystal orientations relative to the beam axis, and its efficiency exceeds $95 \%$, thereby surpassing any previously observed value. These observations suggest new perspectives for the manipulation of high-energy beams, e.g., for collimation and extraction in new-generation hadron colliders, such as the CERN Large Hadron Collider.
\end{abstract}

DOI: 10.1103/PhysRevLett.98.154801

PACS numbers: 29.27.- a, 42.79.Ag, 61.85.+p

Volume reflection of charged particles in bent crystals was theoretically predicted by Taratin and Vorobiev [1] and recently observed in experiments with 70 [2] and $1 \mathrm{GeV}$ [3] protons. The effect should play an essential role in the interaction of a circulating beam with a bent crystal [4] and, under certain conditions, could be used for beam extraction and collimation [5]. Possible evidence of this effect from crystal collimation experiments at the Relativ- 
istic Heavy Ion Collider and Tevatron has been conjectured recently [6].

In this Letter, we report the study of the volume reflection effect with an ultrarelativistic proton beam. The study was undertaken to provide a base for the development of a crystal collimation system for the CERN Large Hadron Collider in order to reach its highest luminosity. Another goal of the study was to extend the investigations made at the Institute for High Energy Physics (IHEP) with strip crystals [7] and more recently at IHEP and the Petersburg Nuclear Physics Institute with quasimosaic silicon crystals successfully used in the channeling mode for the first time [8].

Channeling is the particle confinement between crystalline planes occurring when a particle hits a crystal with momentum nearly parallel to the atomic planes, and its transverse energy does not exceed the depth of the potential well $U_{0}$ between the planes [see Figs. 1(a) and 1(c)]. Channeled particles perform an oscillatory motion with period $\lambda$ that depends on the particle momentum and the planar potential (tens of micrometers for ultrarelativistic protons in silicon lattice). In a bent crystal, a centrifugal term, proportional to the curvature, reduces the potential well. For moderate bending, the potential wells are preserved, and the channeling remains effective [see Fig. 1(b)].

A particle with a large transverse component of the momentum cannot enter the channeling regime at the crystal surface but may arrive at a tangency point with the bent crystallographic planes while crossing the crystal. Here two effects may take place [Fig. 1(b)]: either the (a)

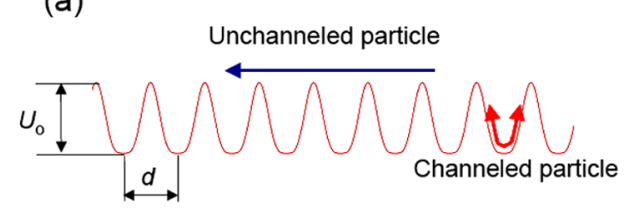

(b)

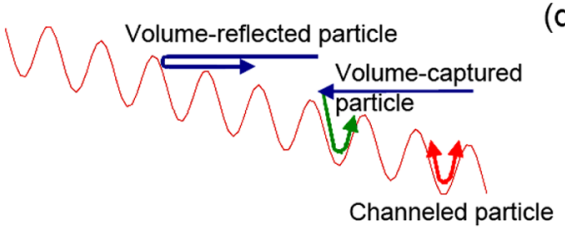

(c)

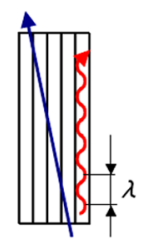

(d)

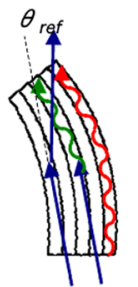

FIG. 1 (color online). (a) Periodic planar potential in a straight crystal for positively charged particles. The arrows show a channeled particle with oscillatory motion in the potential depth and a nonchanneled particle, whose transverse energy is greater than the depth of the potential well $U_{o}$. (c) Schematic representation of the particle trajectories in a straight crystal. (b) Periodic planar potential in a bent crystal for positively charged particles. The arrows show volume-reflected, volume-captured, and channeled particles. (d) Schematic representation of the particle trajectories in a bent crystal. particle partially loses its transverse energy and gets trapped into the channel (volume capture), or its transverse direction is elastically reversed by the interaction with the potential barrier (volume reflection).

Volume capture scales with the particle energy $E$ approximately as $E^{-3 / 2}$ and thereby is less probable at high energies where volume reflection becomes the dominant effect. Almost all particles are then subject to volume reflection, resulting in a transverse kick that deflects them externally with respect to the center of curvature of the crystalline planes [Fig. 1(d)]. Numerical simulations predict that relativistic protons interacting with a bent silicon crystal may be reflected with a deflection angle up to 1.5 times the critical angle $\theta_{c}=\left(2 U_{o} / \mathrm{pc}\right)^{1 / 2}$.

An experiment was carried out with a $400 \mathrm{GeV}$ proton beam from the CERN Super Proton Synchrotron H8 external line, which had a low divergence and an intensity near $10^{4}$ particles per second.

A sketch of the experimental layout is shown in Fig. 2(c). It consisted of a high precision goniometer $(\mathrm{G})$, where the crystals under investigation were mounted, and of various detectors to track particles. They were positioned along the beam line in the vicinity of the crystal and in an experimental area at about $70 \mathrm{~m}$ downstream.

For the experiment, we prepared both quasimosaic and striplike silicon crystals fabricated according to the technologies developed in Refs. [8,9], respectively. In the case of the quasimosaic crystal, a bending device [Fig. 2(a)] similar to the one described in Ref. [10] was applied. Bending of the striplike crystal was achieved via anticlastic forces in a specially designed holder [Fig. 2(b)] originated from Ref. [11]. Results of measurements with these crystals turned out to be similar. We present here results obtained with a strip crystal and then very briefly compare them to those with a quasimosaic crystal.

The strip crystal had (110) channeling planes bent at an angle of $162 \mu \mathrm{rad}$ along its $3 \mathrm{~mm}$ length in the beam direction. The planar potential for (110) planes is $U_{0}=$ $22.7 \mathrm{eV}$ and corresponds to a critical angle of $10.6 \mu \mathrm{rad}$. The multiple scattering angle of $400 \mathrm{GeV}$ protons in this crystal is equal to $5.3 \mu \mathrm{rad}$ and, therefore, is small with respect to the critical angle.

The $\mathrm{H} 8$ beam line was adjusted to provide a divergence of $(8 \pm 1) \mu \mathrm{rad}$, smaller than the critical angle. The beam spot size was of about $1 \mathrm{~mm}$, i.e., of the same order of the strip crystal thickness.

The goniometer consists of three high precision motion units, two linear and one angular. With the linear motions, the crystals were positioned with respect to the beam center with an accuracy of several micrometers within a range of about $10 \mathrm{~cm}$. With angular scans, the crystals were aligned with respect to the beam axis with an accuracy of $1.5 \mu \mathrm{rad}$ within the full turn. To increase its mechanical stability, the goniometer was placed on a special granite table. 


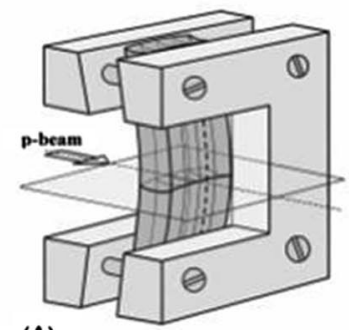

(A)

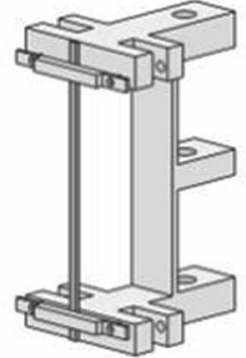

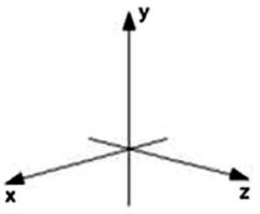

(B)

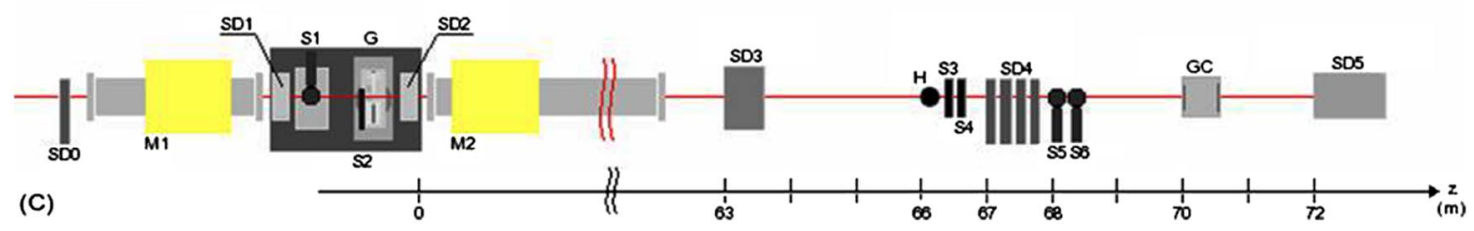

FIG. 2 (color online). (a),(b) Two different types of holders to host and bend crystals. (c) Schematic drawing of the H8-RD22 experimental setup. M1 and M2 are two bending magnets, part of the H8 beam transport line with no specific function for this experiment. See the text for more details.

Six scintillator counters were placed at different positions to determine the beam transverse offset with respect to the crystal (S1-S2), to provide the basic trigger signal for silicon detectors (S3-S4), and to measure the beam divergence and the beam profile (S5-S6).

A scintillation hodoscope $(\mathrm{H})$ and a position-sensitive gas chamber (GC) able to sustain high particle fluxes were operated in self-triggering mode for a fast prealignment of the crystal. High statistical runs were taken instead with a set of silicon microstrip detectors ( $\mathrm{SD} n$ ) of spatial resolution in the range 10-30 $\mu \mathrm{m}[12,13]$ placed both upstream $(-0.5$ and $-6 \mathrm{~m})$ and downstream $(0.5,64.8,69$, and $74 \mathrm{~m})$ with respect to the crystal position.

Figure 3 shows a summary plot of a scan performed with the strip crystal. The horizontal angular deflection of the particles measured at $64.8 \mathrm{~m}$ downstream is registered on the $x$ axis of the plot, while the crystal orientation angle is on the $y$ axis. Six distinct regions of interest are visible: in region (1), the beam "sees" the crystal as an amorphous material and no peculiar effects can be observed. In region (2), channeling is effective at an angle of (165 \pm 2) $\mu \mathrm{rad}$, in agreement with the value of the bending angle of the crystal. The measured fraction of incoming particles deflected by the crystal through channeling in region (2) is about $55 \%$. Events, in which the particle escapes from the channels along the crystal length (dechanneling), fill the dim region (3). At larger angles, channeling at the entry face of the crystal is no longer possible, though, but in region (4) the beam clearly appears to be deflected in a direction opposite to that of channeling. This is the region where proton volume reflection occurs. A Gaussian fit to the reflected beam distribution yields a deflection angle $\theta_{\mathrm{vr}}=[13.9 \pm 0.2$ (statistical) \pm 1.5 (systematic) $] \mu \mathrm{rad}$, which is in agreement with the prediction of analytical calculation $(14.5 \mu \mathrm{rad})$ and of Monte Carlo simulation 14.7-15.0 $\mu \mathrm{rad}$ [14]. The details of simulation and com- parison with data of the tested crystals will be the subject of future publications. The current experimental uncertainty originated from limited statistics and from the interplay of the uncertainty on the impact point position and the crystal shape distortion in the vertical plane.

The extension of region (4) along the $y$ axis is comparable with the bending angle of the crystal. Such evidence confirms that the shift of the beam profile in region (4) is due to volume reflection. Moreover, such a range is much wider than for channeling.

Region (5) corresponds to particles, which, due to multiple scattering on the crystal nuclei and electrons, are

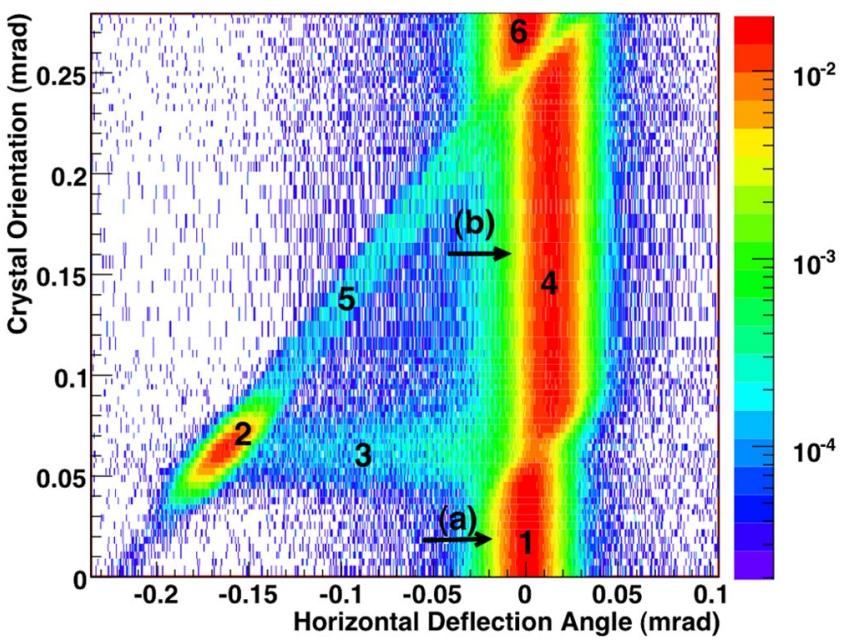

FIG. 3 (color online). Beam intensity recorded by the silicon microstrip detectors as a function of the horizontal deflection angle ( $x$ axis) and the crystal orientation ( $y$ axis). Six regions can be distinguished: (1) and (6) nonchanneling mode; (2) channeling; (3) dechanneling; (4) volume reflection; (5) volume capture. The wider angular acceptance of volume reflection compared to channeling is clearly visible in the figure. 


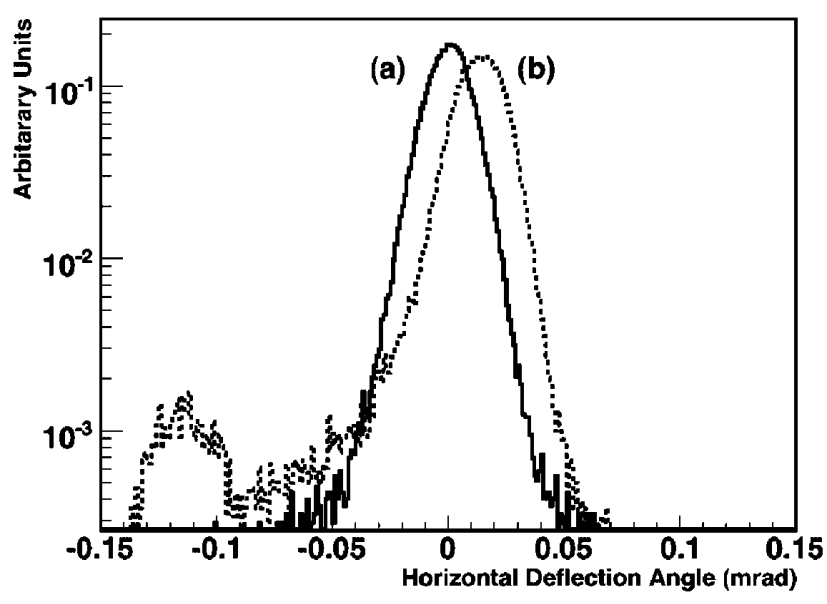

FIG. 4. Beam profile for (a) nonchanneling and (b) volume reflection modes; the profiles correspond to the crystal orientation shown in Fig. 3 and exhibit very similar shapes.

captured in the channeling regime at an intermediate point inside the crystal volume, hence undergoing a lower deflection.

As the crystal orientation angle increases beyond the bending angle in region (6), the beam sees the crystal again as an amorphous layer with similar features as in region (1).

Analysis of the beam profile for two different crystal orientations, one in region (1) and one in region (4) (see Fig. 4), demonstrates that volume reflection does not appreciably alter the profile of the beam.

The volume reflection is a crucial finding for beam steering. In order to quantify this capability, an important parameter is the reflection efficiency, namely, the fraction of particles being volume reflected. A particle is considered as reflected when its angle with the incident beam axis is $\theta_{\text {def }}>\theta_{\mathrm{vr}}-3 \sigma_{\mathrm{vr}}$, where $\sigma_{\mathrm{vr}}$ is the rms deviation for the peak of the reflected beam. From a preliminary analysis of the profiles in region (4), such as those shown in Fig. 4, the volume reflection efficiency has been estimated to exceed $95 \%$. This high efficiency is by far greater than the maximal theoretical single-pass efficiency for channeling. A detailed analysis is in progress to precisely measure such efficiency values for reflection and for channeling. It will make use of the upstream detectors to determine the beam fraction effectively seen by the crystal.

We also measured the volume reflection effect with a quasimosaic silicon crystal. This crystal had been bent along (111) crystallographic planes, its length and bend angle being equal to $0.84 \mathrm{~mm}$ and $72 \mu \mathrm{rad}$, respectively. The measured deflection angle of the volume-reflected beam was near $13 \mu \mathrm{rad}$ and the reflection efficiency close to the strip crystal case. A difference between the crystals agrees within the margin of experimental errors reported above.
In conclusion, volume reflection exhibits superior performance compared to channeling in terms of efficiency and angular acceptance. Volume reflection sheds new insight into the physics of interaction of charged particles with crystals and suggests new applications. Manipulation of a high-energy beam via volume reflection appears to be a viable technique in the next generation of experiments in both accelerator and high-energy physics. As an example, a short bent crystal may be employed as a smart deflector to aid halo collimation in high-intensity hadron colliders or as a device to separate low-angle scattering events in diffractive physics.

We gratefully acknowledge support from Lau Gatignon, Ilias Efthymiopoulos, P. Lebrun, S. Myers, Alexei A. Vorobyev, Peter M. Levchenko, Alexei N. Sissakian, Alexander I. Malakhov, Nikolai E. Tyurin, S. Chiozzi, A. Sambo, E. Boscolo Marchi, A. Papi, V. Postolache, and G. Alberti. We also acknowledge partial support by the European Community-Research Infrastructure Activity under the FP6 "Structuring the European Research Area" program (CARE, Contract No. RII3-CT-2003-506395), the INTAS program and MIUR Project No. 2006028442, Russian Foundation for Basic Research Grant No. 06-0216912, Council of the President of the Russian Federation Grant No. NSh-3057.2006.2, and the "Physics of Elementary Particles and Fundamental Nuclear Physics " program of the Russian Academy of Sciences.

[1] A. M. Taratin and S. A. Vorobiev, Phys. Lett. A 119, 425 (1987); Nucl. Instrum. Methods Phys. Res., Sect. B 26, 512 (1987).

[2] Y. M. Ivanov et al., Phys. Rev. Lett. 97, 144801 (2006).

[3] Y. M. Ivanov et al., JETP Lett. 84, 372 (2006).

[4] A. M. Taratin, Nucl. Instrum. Methods Phys. Res., Sect. B 95, 243 (1995).

[5] I. A. Yazynin, in Proceedings of the 1995 Particle Accelerator Conference (IEEE, New York, 1996), Vol. 3, pp. 1952-1954.

[6] R. A. Carrigan et al., FERMILAB Report No. FERMILABCONF-06-309-AD, 2006.

[7] A. G. Afonin et al. Phys. Rev. Lett. 87, 094802 (2001).

[8] Y.M. Ivanov, A. A. Petrunin, and V. V. Skorobogatov, JETP Lett. 81, 99 (2005).

[9] V. Guidi et al., Nucl. Instrum. Methods Phys. Res., Sect. B 234, 40 (2005).

[10] Y. M. Ivanov, CERN Report No. CC-2005 CARE-HHHAPD, 2005.

[11] A. G. Afonin et al., JETP Lett. 67, 781 (1998).

[12] M. Prest et al., Nucl. Instrum. Methods Phys. Res., Sect. A 501, 280 (2003).

[13] B. Alpat et al., Nucl. Instrum. Methods Phys. Res., Sect. A 439, 53 (2000).

[14] H8-RD22 Collaboration (unpublished). 